ISSN 0258-7122

Bangladesh J. Agril. Res. 33(3) : 647-654, December 2008

\title{
INTEGRATED WEED MANAGEMENT IN POTATO AT MUNSHIGONJ
}

\author{
A.S.A. KHAN ${ }^{1}$, M.A. HOSSAIN ${ }^{2}$, A.A. MAHMUD ${ }^{3}$, \\ M.I.A. HOWLADER ${ }^{4}$ AND M.A. RAHMAN ${ }^{5}$
}

\begin{abstract}
A field experiment was carried out at the research farm of Tuber Crops Research Sub-station, Munshigonj of Bangladesh Agricultural Research Institute (BARI) during rabi seasons of 2005-06 and 2006-07 to find out the effective and economical integrated weed control method in potato. Chenopodium album and Amaranthus viridis were the major weed species in potato field. Mulch (water hyacinth) along with herbicide application (Ronstar 25 EC @ $1 \mathrm{ml} / \mathrm{L}$ water) at 7 days after planting (DAP) was found most effective in controlling weed population (94 to 95\%) upto 30 days of planting than that employed by only mulch (45 to 53\%). The weeds were found to reduce tuber yield to the extent of 43 percent. Mulching plus herbicide spraying at 7 DAP followed by one time uprooting of weeds by hand at 25 DAP produced the highest tuber yield of 23.39 t/ha in 2005-06 and 29.58 t/ha in 2006-07 and it was most effective in controlling weeds as compared to unweeded and mulched plots. The highest net returns (Tk. 116141/ha in 2005-06 and Tk. 205646/ha in 2006-07) and maximum benefit cost ratio of 2.64 and 3.28 were noted with mulching by water hyacinth + herbicide application at $7 \mathrm{DAP}+$ one time weed uprooting by hand at 25 DAP.
\end{abstract}

Key Words: Weed management, mulching, herbicide, potato tuber yield.

\section{Introduction}

Potato (Solanum tuberosum) is one of the major crops cultivated during rabi season as popular vegetables in Bangladesh. Munshigonj area is one of the major contributors in national potato production. This location covered an area of 32,170 ha in potato cultivation (Anon., 2007). Intense weed competition is one of the constraints in realizing potential yield of potato resulting in substantial reduction in the yield (Singh et al., 1984). Weeds deplete crops environment of nutrients, water and light, thus yields of potato is reduced considerably. In addition, weeds cause spread of several diseases and pests. The quality of produce is also reduced by weed infestation (Pandey, 2000). However, farmers are controlling weeds by hand weeding in several times. Manual weeding is costly, time consuming and sometimes not possible due to non-availability of labour. In the studied area, (Munshigonj), scarcity of day labourer is found during crop season and labourer hikes more charges that increases cost of production. In this situation, farmers of

${ }^{1,2 \& 5}$ Agronomy Division, BARI, Joydebpur, ${ }^{3}$ Tuber Crops Research Sub-station, BARI, Munshiganonj, ${ }^{4}$ On-Farm Research Division, BARI, Patuakhali, Bangladesh. 
Munshigaonj area usually needs a profitable technology for weed management practices that may solve their problem. Hence, the present study was undertaken to find out an effective and economic weed control method in potato cultivation.

\section{Materials and Method}

The experiment was conducted at the research farm of Tuber Crops Research Sub-station, Munshigonj of Bangladesh Agricultural Research Institute (BARI) during rabi seasons of 2005-06 and 2006-07, respectively. The land type was medium high and the Soil was silty clay in texture, which belongs to old Meghna Estuarine Floodplain soil (AEZ-19). Seven weed management practices including one untreated control were used as treatment variables. The treatments were Untreated control (weedy check)- $T_{1}$, Mulching by water hyacinth- $T_{2}$, Mulching + one time weed uprooting by hand at 25 and 45 DAP- $\mathrm{T}_{4}$, Mulching + herbicide (Ronstar 25 EC @ 1ml/L water) spray at 7 DAP as post planting post emergence weed control- $\mathrm{T}_{5}$, Mulching + herbicide (Ronstar 25 EC @ 1ml/L water) spray at $7 \mathrm{DAP}+$ weed uprooting at $25 \mathrm{DAP}-\mathrm{T}_{6}$, Weed free (weeding was done manually at 20, 40, 60 and $80 \mathrm{DAP}$ )- $\mathrm{T}_{7}$. The experiment was laid out in a randomized complete block design with three replications. The unit plot size was $4 \mathrm{~m} \times 3 \mathrm{~m}$. The potato variety Diamant was used as the test crop. The potato whole tubers were planted on 27 November in 2005 and 23 November in 2006 at a spacing of $60 \mathrm{~cm} \times 25 \mathrm{~cm}$. The crop was fertilized with $150-43-140-22 \mathrm{~kg}$ NPKS/ha (FRG, 2005). During the final land preparation, full dose of P, K, S and $50 \% \mathrm{~N}$ were applied as basal. Remaining $\mathrm{N}$ was applied at 30 days after planting as side dressed. Herbicide was applied at 7 DAP through Knapsack sprayer using 100 liter water/ha. Irrigation was applied two times each at 30 and 55 DAP only in furrows. Admire 20 EC was sprayed @ 1ml/L of water at 25 and 50 DAP to prevent insect pests. Dithane M-45 @ 2g/L of water was sprayed at 45 and 60 DAP as preventive measure of diseases. No rainfall was occurred in 2005-06, but crop received $50 \mathrm{~mm}$ rainfall in 2006-07. Data on weed population was recorded at 30 DAP from a quadrate of $0.5 \mathrm{~m}$ placed randomly at 2 places in each plot and their biomass were taken at 90 DAP. Weed control efficiency (WCE) was calculated by using the formula: WCE $=$ (weed biomass in unweeded control weed biomass in managed treatment)/ weed biomass in unweeded control $\times 100$. Data on weed density and weed biomass were transformed by square root transformation method before conductiong analysis variance. Cost benefit analysis was done according to prevailing market price.

\section{Results and Discussion}

\section{Weed species and infestations}

Weed infestations in number of different weed species as affected by different weed management practices at 30 DAP are presented in Table 1. The major 
Table 1. Effect of water hyacinth mulch and Ronstar 25 EC in controlling weeds after 30 days of palnting at Munshigonj during 2005-06 and 2006-027.

\begin{tabular}{|c|c|c|c|c|c|c|c|c|c|c|c|c|c|c|}
\hline \multirow{3}{*}{ Treatments } & \multicolumn{14}{|c|}{ Control of weed species over no weeding (\%) } \\
\hline & \multicolumn{2}{|c|}{$\begin{array}{l}\text { Chenopodium } \\
\text { album }\end{array}$} & \multicolumn{2}{|c|}{$\begin{array}{c}\text { Amaranthus } \\
\text { viridis }\end{array}$} & \multicolumn{2}{|c|}{$\begin{array}{l}\text { Alternanthera } \\
\text { philoxeroides }\end{array}$} & \multicolumn{2}{|c|}{$\begin{array}{l}\text { Eleusine } \\
\text { indica }\end{array}$} & \multicolumn{2}{|c|}{$\begin{array}{l}\text { Cyperus } \\
\text { rotundus }\end{array}$} & \multicolumn{2}{|c|}{$\begin{array}{l}\text { Glinus } \\
\text { lotoides }\end{array}$} & \multicolumn{2}{|c|}{ Total } \\
\hline & 05-06 & $06-07$ & $05-06$ & $06-07$ & $05-06$ & $06-07$ & $05-06$ & $06-07$ & $05-06$ & $06-07$ & $05-06$ & $06-07$ & $05-06$ & $06-07$ \\
\hline $\mathrm{T}_{1}$ & $\begin{array}{c}0 \\
(340)\end{array}$ & $\begin{array}{c}0 \\
(340)\end{array}$ & $\begin{array}{c}0 \\
(32)\end{array}$ & $\begin{array}{c}0 \\
(32)\end{array}$ & $\begin{array}{c}0 \\
(4)\end{array}$ & $\begin{array}{c}0 \\
(4)\end{array}$ & $\begin{array}{c}0 \\
(8)\end{array}$ & $\begin{array}{c}0 \\
(8)\end{array}$ & $\begin{array}{c}0 \\
(1)\end{array}$ & $\begin{array}{c}0 \\
(1)\end{array}$ & $\begin{array}{c}0 \\
(1)\end{array}$ & $\begin{array}{c}0 \\
(1)\end{array}$ & $\begin{array}{c}0 \\
(387)\end{array}$ & $\begin{array}{c}0 \\
(387)\end{array}$ \\
\hline $\mathrm{T}_{2}$ & $\begin{array}{c}59 \\
(140)\end{array}$ & $\begin{array}{c}59 \\
(140)\end{array}$ & $\begin{array}{c}50 \\
(16)\end{array}$ & $\begin{array}{c}50 \\
(16)\end{array}$ & $\begin{array}{l}-300 \\
(16)\end{array}$ & $\begin{array}{l}-300 \\
(16)\end{array}$ & $\begin{array}{l}50 \\
(4)\end{array}$ & $\begin{array}{l}50 \\
(4)\end{array}$ & $\begin{array}{c}-300 \\
(4)\end{array}$ & $\begin{array}{c}-300 \\
(4)\end{array}$ & $\begin{array}{c}-300 \\
(4)\end{array}$ & $\begin{array}{c}-300 \\
(4)\end{array}$ & $\begin{array}{c}53 \\
(184)\end{array}$ & $\begin{array}{c}53 \\
(184)\end{array}$ \\
\hline $\mathrm{T}_{3}$ & $\begin{array}{l}100 \\
(0)\end{array}$ & $\begin{array}{c}100 \\
(0)\end{array}$ & $\begin{array}{l}100 \\
(0)\end{array}$ & $\begin{array}{l}100 \\
(0)\end{array}$ & $\begin{array}{l}100 \\
(0)\end{array}$ & $\begin{array}{l}100 \\
(0)\end{array}$ & $\begin{array}{l}100 \\
(0)\end{array}$ & $\begin{array}{c}100 \\
(0)\end{array}$ & $\begin{array}{l}100 \\
(0)\end{array}$ & $\begin{array}{l}100 \\
(0)\end{array}$ & $\begin{array}{l}100 \\
(0)\end{array}$ & $\begin{array}{l}100 \\
(0)\end{array}$ & $\begin{array}{l}100 \\
(0)\end{array}$ & $\begin{array}{l}100 \\
(0)\end{array}$ \\
\hline $\mathrm{T}_{4}$ & $\begin{array}{l}100 \\
(0)\end{array}$ & $\begin{array}{l}100 \\
(0)\end{array}$ & $\begin{array}{l}100 \\
(0)\end{array}$ & $\begin{array}{l}100 \\
(0)\end{array}$ & $\begin{array}{l}100 \\
(0)\end{array}$ & $\begin{array}{l}100 \\
(0)\end{array}$ & $\begin{array}{l}100 \\
(0)\end{array}$ & $\begin{array}{l}100 \\
(0)\end{array}$ & $\begin{array}{l}100 \\
(0)\end{array}$ & $\begin{array}{l}100 \\
(0)\end{array}$ & $\begin{array}{l}100 \\
(0)\end{array}$ & $\begin{array}{l}100 \\
(0)\end{array}$ & $\begin{array}{l}100 \\
(0)\end{array}$ & $\begin{array}{l}100 \\
(0)\end{array}$ \\
\hline $\mathrm{T}_{5}$ & $\begin{array}{c}96 \\
(12)\end{array}$ & $\begin{array}{c}96 \\
(12)\end{array}$ & $\begin{array}{l}88 \\
(4)\end{array}$ & $\begin{array}{l}88 \\
(4)\end{array}$ & $\begin{array}{l}100 \\
(0)\end{array}$ & $\begin{array}{l}100 \\
(0)\end{array}$ & $\begin{array}{l}100 \\
(0)\end{array}$ & $\begin{array}{l}100 \\
(0)\end{array}$ & $\begin{array}{c}-700 \\
(8)\end{array}$ & $\begin{array}{c}-700 \\
(8)\end{array}$ & $\begin{array}{l}100 \\
(0)\end{array}$ & $\begin{array}{l}100 \\
(0)\end{array}$ & $\begin{array}{c}94 \\
(24)\end{array}$ & $\begin{array}{c}94 \\
(24)\end{array}$ \\
\hline $\mathrm{T}_{6}$ & $\begin{array}{l}100 \\
(0)\end{array}$ & $\begin{array}{l}100 \\
(0)\end{array}$ & $\begin{array}{l}100 \\
(0)\end{array}$ & $\begin{array}{l}100 \\
(0)\end{array}$ & $\begin{array}{c}100 \\
(0)\end{array}$ & $\begin{array}{l}100 \\
(0)\end{array}$ & $\begin{array}{c}100 \\
(0)\end{array}$ & $\begin{array}{c}100 \\
(0)\end{array}$ & $\begin{array}{c}100 \\
(0)\end{array}$ & $\begin{array}{c}100 \\
(0)\end{array}$ & $\begin{array}{l}100 \\
(0)\end{array}$ & $\begin{array}{c}100 \\
(0)\end{array}$ & $\begin{array}{c}100 \\
(0)\end{array}$ & $\begin{array}{l}100 \\
(0)\end{array}$ \\
\hline $\mathrm{T}_{7}$ & $\begin{array}{l}100 \\
(0)\end{array}$ & $\begin{array}{l}100 \\
(0)\end{array}$ & $\begin{array}{l}100 \\
(0)\end{array}$ & $\begin{array}{l}100 \\
(0)\end{array}$ & $\begin{array}{l}100 \\
(0)\end{array}$ & $\begin{array}{l}100 \\
(0)\end{array}$ & $\begin{array}{l}100 \\
(0)\end{array}$ & $\begin{array}{l}100 \\
(0)\end{array}$ & $\begin{array}{l}100 \\
(0)\end{array}$ & $\begin{array}{l}100 \\
(0)\end{array}$ & $\begin{array}{l}100 \\
(0)\end{array}$ & $\begin{array}{l}100 \\
(0)\end{array}$ & $\begin{array}{l}100 \\
(0)\end{array}$ & $\begin{array}{l}100 \\
(0)\end{array}$ \\
\hline
\end{tabular}

* Number of weed species $/ \mathrm{m}^{2}$ area are presented in parentheses.

Here,

$\mathrm{T}_{1}=$ Untreated control, $\mathrm{T}_{2}=$ Mulching, $\mathrm{T}_{3}=$ Mulching + one time weed uprooting by hand at 25 DAP, $\mathrm{T}_{4}=$ Mulching + tow times weed uprooting by hand at 25 and 45 DAP, $\mathrm{T}_{5}=$ Mulching + Ronstar at 7 DAP, $\mathrm{T}_{6}=$ Mulching + Ronstar at 7 DAP + one time weed uprooting by hand at $25 \mathrm{DAP}$ and $\mathrm{T}_{7}=$ Weed free. 
weeds infested the crop in the experimental field were Chenopodium album (bathua), Amaranthus viridis (shaknotey), Alternanthera philoxeroides (maloncho). Among the weed species, Chenopodium album (bathua) and Amaranthus viridis (shaknotey) were the dominant. The results are in agreement with the findings of Anon. (2005) Weed management methods like mulching, mulching plus application of herbicide was the most effective in controlling weed populations over control upto 30 DAP. Among the methods, mulching plus application of herbicide Ronstar 25 EC @ 1ml/L water at 7 DAP was much more effective in suppressing weeds than planted by mulching only. Application of Ronstar 25 EC controlled weeds by 94\% during 2006 and 95\% during 2007, while only mulching treated plots suppressed weeds 53\% in 2005-06 and $45 \%$ in 2006-07.

\section{Weed density}

Weed density was significantly influenced by different weed management practices (Table 2). The highest weed density 349 and $373 / \mathrm{m}^{2}$ were recorded in untreated control plot in 2005-06 and 2006-07, respectively, and it was followed by only mulching plots $\left(261 / \mathrm{m}^{2}\right.$ in $2005-06$ and $257 / \mathrm{m}^{2}$ in 2006-07). The weed density in mulching plus 1 hand weeding at $25 \mathrm{DAP}$ and mulching plus herbicide spraying at 7 DAP were statistically indentical. The lowest weed density (56 and $73 / \mathrm{m}^{2}$ ) were recorded from the plots where mulching plus uprooting of weeds by hand were done at 25 and 45 DAP in both the years and it was statistically identical with mulching plus herbicide spraying followed by uprooting of weeds by hand.

\section{Weed biomass}

Weed fresh biomass was significantly influenced by different weed management practices (Table 2). The highest weed fresh biomasses of $1177 \mathrm{~g} / \mathrm{m}^{2}$ and $1467 \mathrm{~g} / \mathrm{m}^{2}$ were obtained from untreated control in 2005-06 and 2006-07, respectively. Though, it did not significantly differ from mulch treated plots in 2005-06 but differed significantly $\left(1017 \mathrm{~g} / \mathrm{g}^{2}\right)$ in 2006-07. The lowest weed biomass 210 and $287 \mathrm{~g} / \mathrm{m}^{2}$ were recorded from mulching plus two uprooting of weeds by hand and it was statistically identical with the weed biomass from mulching along with application of herbicide Ronstar 25 EC followed by one time uprooting of weeds by hand.

\section{Weed control efficiency}

The weed control efficiency among the weed management practices ranged from 18 to 100 in 2005-06 and 31 to $100 \%$ in 2006-07 (Table 2). The highest weed control efficiency was found in weed free plots followed by mulching plus two times uprooting of weeds by hand (80 to $82 \%$ ), mulching plus herbicide spraying (64 to $76 \%$ ). The lowest weed control efficiency (18 to 31\%) was recorded where only mulch was used. Similar results were also observed by Jaiswal and Lal (1996). 
Table 2. Effect of weed management practices on weed density, weed biomass (fresh) and weed control efficiency at 90 days after planting (DAP) of potato tuber during 2005-06 and 2006-07.

\begin{tabular}{|c|c|c|c|c|c|c|}
\hline \multirow[t]{2}{*}{ Treatments } & \multicolumn{2}{|c|}{ Weed density (no./m²) } & \multicolumn{2}{|c|}{ Weed biomass $\left(\mathrm{g} / \mathrm{m}^{2}\right)$} & \multicolumn{2}{|c|}{ Weed control efficiency (\%) } \\
\hline & 2005-06 & 2006-07 & 2005-06 & 2006-07 & 2005-06 & 2006-07 \\
\hline Untreated control & $\begin{array}{l}18.36 \\
(349)\end{array}$ & $\begin{array}{l}19.30 \\
(373)\end{array}$ & $\begin{array}{c}34.15 \\
(1177)\end{array}$ & $\begin{array}{c}38.27 \\
(1467)\end{array}$ & - & - \\
\hline Mulching & $\begin{array}{l}17.01 \\
(261)\end{array}$ & $\begin{array}{l}15.90 \\
(257)\end{array}$ & $\begin{array}{l}31.08 \\
(967)\end{array}$ & $\begin{array}{c}31.86 \\
(1017)\end{array}$ & 18 & 31 \\
\hline $\begin{array}{l}\text { Mulching + one time weed uprooting } \\
\text { at } 25 \text { DAP }\end{array}$ & $\begin{array}{l}10.97 \\
(121)\end{array}$ & $\begin{array}{l}12.10 \\
(147)\end{array}$ & $\begin{array}{l}18.14 \\
(330)\end{array}$ & $\begin{array}{l}23.63 \\
(558)\end{array}$ & 72 & 62 \\
\hline $\begin{array}{l}\text { Mulching }+ \text { two times weed } \\
\text { uprooting Ronstar at } 7 \text { DAP }\end{array}$ & $\begin{array}{l}7.50 \\
(56)\end{array}$ & $\begin{array}{l}8.53 \\
(73)\end{array}$ & $\begin{array}{l}14.44 \\
(210)\end{array}$ & $\begin{array}{l}16.93 \\
(287)\end{array}$ & 82 & 80 \\
\hline $\begin{array}{l}\text { Mulching + Ronstar at } 7 \mathrm{DAP}+\text { one } \\
\text { time weed uprooting at } 25 \mathrm{DAP}\end{array}$ & $\begin{array}{l}9.63 \\
(94)\end{array}$ & $\begin{array}{l}9.23 \\
(88)\end{array}$ & $\begin{array}{l}16.27 \\
(267)\end{array}$ & $\begin{array}{l}17.77 \\
(317)\end{array}$ & 77 & 78 \\
\hline Weed free & $\begin{array}{l}0.77 \\
(0)\end{array}$ & $\begin{array}{c}0.71 \\
(0)\end{array}$ & $\begin{array}{c}0.71 \\
(0)\end{array}$ & $\begin{array}{c}0.71 \\
(0)\end{array}$ & 100 & 100 \\
\hline LSD (0.05) & 1.77 & 2.47 & 3.47 & 2.58 & - & - \\
\hline CV (\%) & 9.24 & 12.69 & 10.37 & 6.67 & - & - \\
\hline
\end{tabular}

* Data on weed density and weed biomass were transformed by square root transformation method before conduction analysis of variance. Actual values are given in parentheses. 
Table 3. Yield, cost and benefit analysis of potato as affected by different weed management practices during 2005-06 and 200607.

\begin{tabular}{c|ccc|c|c|c|c|c|c|c}
\hline \multirow{2}{*}{ Treatments } & \multicolumn{2}{|c|}{ Tuber yield (t/ha) } & \multicolumn{2}{c|}{ Gross return (Tk/ha) } & \multicolumn{2}{c|}{ Total cost (Tk/ha) } & \multicolumn{2}{c|}{ Net return (Tk/ha) } & \multicolumn{2}{c}{ DCR } \\
\cline { 2 - 11 } & $2005-06$ & $2006-07$ & $2005-06$ & $2006-07$ & $2005-06$ & $2006-07$ & $2005-06$ & $2006-07$ & $2005-06$ & $2006-07$ \\
\hline $\mathrm{T}_{1}$ & 13.78 & 13.30 & 110240 & 133000 & 63568 & 80556 & 46672 & 52444 & 1.73 & 1.65 \\
$\mathrm{~T}_{2}$ & 21.07 & 24.36 & 168560 & 243600 & 68899 & 86142 & 99661 & 157458 & 2.45 & 2.83 \\
$\mathrm{~T}_{3}$ & 22.26 & 27.88 & 178080 & 278800 & 70579 & 89390 & 107501 & 189410 & 2.52 & 3.12 \\
$\mathrm{~T}_{4}$ & 22.89 & 28.55 & 183120 & 285500 & 71699 & 91294 & 111421 & 194206 & 2.55 & 3.13 \\
$\mathrm{~T}_{5}$ & 22.93 & 28.05 & 183440 & 280500 & 70139 & 87466 & 113301 & 193034 & 2.62 & 3.21 \\
$\mathrm{~T}_{6}$ & 23.39 & 29.58 & 187120 & 295800 & 70979 & 90154 & 116141 & 205646 & 2.64 & 3.28 \\
$\mathrm{~T}_{7}$ & 22.46 & 25.21 & 179680 & 252100 & 68608 & 88620 & 111072 & 163480 & 2.62 & 2.84 \\
\hline LSD (0.05) & 2.33 & 5.31 & - & - & - & - & - & - & - & - \\
CV (\%) & 8.05 & 11.825 & - & - & - & - & - & - & - \\
\hline
\end{tabular}

NB. Labour wages @ Tk. 70 in 2005-06 and Tk. 112 in 2006-07

Here,

$\mathrm{T}_{1}=$ Untreated control, $\mathrm{T}_{2}=$ Mulching, $\mathrm{T}_{3}=$ Mulching + one time weed uprooting by hand at 25 DAP, $\mathrm{T}_{4}=$ Mulching + tow times weed uprooting by hand at 25 and $45 \mathrm{DAP}, \mathrm{T}_{5}=$ Mulching + Ronstar at $7 \mathrm{DAP}, \mathrm{T}_{6}=$ Mulching + Ronstar at 7 DAP + one time weed uprooting by hand at 25 DAP and $T_{7}=$ Weed free. 


\section{Tuber yield}

Tuber yield of potato was significantly influenced due to different weed management practices (Table 3). Untreated control treatment gave the lowest tuber yield of $13.78 \mathrm{t} / \mathrm{ha}$ in 2005-06 and 13.30 t/ha in 2006-07. The treatment mulching plus herbicide spraying followed by one time uprooting of weeds by hand produced the maximum yields of 23.39 t/ha in 2005-06 and 29.58 t/ha in 2006-07 and it was statistically identical with mulching plus herbicide spraying mulching plus two times weed uprooting by hand, mulching plus one time weed uprooting by hand, weed free plots and only mulching treatment. Among the two years, higher tuber yield was recorded in 2006-07. Crop received $50 \mathrm{~mm}$ rainfall in 2006-07 during the time of tuber growth that might help in obtaining higher yield than 2005-06. Averaged over the years, weed condition resulted 43\% reduction in tuber yield. Similar results were also reported by Jaiswal and Lal (1996); Borude et al. (2001). Though weed free treatment showed $100 \%$ weed control efficiency but yielded lower than mulching plus two times weed uprooting by hand, mulching plus herbicide spraying and mulching plus herbicide spraying followed by one time weed uprooting by hand at 25 DAP. Water hyacinth might help conserve soil moisture (Wofford and Orzolek, 1993; Lal and Grewal, 1991) and served as an effective barrier to weed emergence.

\section{Cost benefit analysis}

Economic analysis of different weed management practices on potato production was done (Table 3). Maximum gross return of Tk. 187120/ha and 295800/ha were obtained from mulching with application of herbicide Ronstar 25 EC @ $1 \mathrm{~m} / \mathrm{L}$ water followed by one time weed uprooting by hand at $25 \mathrm{DAP}\left(\mathrm{T}_{6}\right)$ in both the years, but it was slightly higher from $T_{5}$ in 2005-06. Similar trend was followed in case of net return. Higher cost was also recorded in treatment $T_{4}$ in both the years. The cost of cultivation was much higher in 2006-07 than in 200506 due to higher labour wages. The maximum benefit cost ratios of 2.64 in 200506 and 3.28 in 2006-07 were obtained from mulching plus herbicide spraying followed by one time weed uprooting by hand at 25 DAP $\left(T_{6}\right)$ and this was followed by mulching plus herbicide spraying at DAP $\left(T_{5}\right)$.

\section{Conclusion}

Based on two years findings, mulching (water hyacinth) with herbicide application (Ronstar 25 EC @ 1ml/L water) at 7 days after planting as post emergence weed control followed by one time uprooting of weeds by hand at 25 days after transplanting would be economically profitable for obtaining maximum tuber yield of potato in Munshigonj area. Though, mulching yield, this treatment was not economically profitable for potato cultivation in Munshigonj area. Therefore, any generalization of the findings, this sort of the study should be conducted covering wider areas of the country. 


\section{References}

Anonymous. 2007. Sarejamin Protibedon-Munshigaonjer Alo. In: BARI Sangbad (Bengali). BARI, Gazipur, Borsho. 19(1): 1-6.

Anonymous. 2005. Survey and identification of major weed species of different crops. Annual Research Report 2004-05. Agronomy Division, BARI, Gazipur-1701.

Borude, S.S., A.V. Solanke and P.U. Raundal. 2001. Integrated weed management in potato in western Maharashtra. J. Indian Potato Assoc. 28: 271-273

FRG. 2005. Fertilizer Recommendation Guide. Bangladesh Agricultural Research Council, Farmgate, New Airport Road, Dhaka-1215. Soils pub. No. 45.

Jaiswal, V.P. and S.S. Lal. 1996. Weed management in potato. Technical Bulletin. 33. Central Potato Research Institute. Simla. p. 10.

Pandey, A.K. 2000. Weed management in vegetable crop. In: Proceedings of National Training Course on Vegetable Production, held at Indian Institute of Vegetable Research, Varanasi, l.c.p. 132-138.

Singh, G., V.M. Bhan, S.S. Tripathi and D. Singh. 1984. Comparative efficacy of herbicides in potato. Indian J. weed Sci. 16: 1-5.

Wofford, Jr.D.J. and M.D. Orzolek. 1993. No irrigation or weeding for 10 years. American Vegetable Grower. Meister Publishing Company. Ohio. USA. 41: 30-32. 